\title{
„HIER KOMMEN IHRE GRAUEN ZELLEN IN FAHRT“ - PHRASEME IN DER ANZEIGENWERBUNG
}

Phrasemen begegnet man auf Schritt und Tritt, sei es in den Medien, in unterschiedlichen Textsorten, in der mündlichen und schriftlichen Kommunikation oder im privaten Bereich. Wo immer sie auftauchen, erweisen sich Phraseme als beliebtes Stilmittel zur Beschreibung von Objekten und Sachverhalten oder zur attraktiven Gestaltung von diversen Kommentaren und Vorgängen, aber auch von Botschaften. Die Beliebtheit von Phrasemen als begehrtes Gestaltungsmittel in der Werbung wird in zahlreichen Untersuchungen bestätigt (vgl. Hemmi 1994, Balsliemke 1999, Balsliemke 2001, Bass 2003, Janich 2005, Janich 2006, Vesalainen 2007, Majnusz-Stadnik 2014, Rypáčková 2019, Umborg 2020).

Der folgende Beitrag konzentriert sich auf Phraseme in Werbeanzeigen der Printmedien und beschränkt sich auf die referentiellen Phraseme im engeren Sinne. Als Basis dienen 84 Werbeanzeigen aus verschiedenen deutschsprachigen Zeitschriften und Magazinen der letzten fünfzehn Jahre (2006-2021). Alle Bedeutungsparaphrasen sind dem Wörterbuch DUDEN 11 oder der Internetseite redensarten-index.de entnommen. Die einzelnen Phraseme, die in den Werbeanzeigen vorkommen, werden je nach Kontext den einzelnen Phrasem-Bild-Bezügen nach Stöckl (2004) zugeordnet. Im Vordergrund der Untersuchung steht die Beziehung zwischen Text und Bild. In den einzelnen Text-BildAnalysen interessiert uns die Frage, welche semantischen Leistungen die visuellen Bilder in den Werbeanzeigen, in denen Phraseme vorkommen, für die Wahrnehmung und das Verständnis des gesamten Textes erbringen, was anhand einer qualitativen Korpusanalyse interpretiert werden soll, die auf der Methode der Datenerfassung basiert. Gezeigt werden soll, welchen Beitrag Phraseologie in Bild und Text für die Werbung leisten kann.

In Anlehnung an Burger (2015) sind Phraseme solche festen Wortverbindungen, die aus mehr als einem Wort zusammengesetzt und allgemein bekannt sind, über einen längeren 
Zeitraum in einer Sprachgemeinschaft verwendet worden sind und eventuell im übertragenen Sinne gemeint sind. Die charakteristischen Eigenschaften der Phraseme sind demzufolge: Polylexikalität, Festigkeit und Reproduzierbarkeit sowie Idiomatizität. Wenn alle Kriterien erfüllt sind, dann geht es um „Phraseologie im engeren Sinne“, wenn das Kriterium der Idiomatizität fehlt, dann spricht man von der „Phraseologie im weiteren Sinne“. Burger (ebd.: 14f.) zählt zu den Phrasemen im weiteren Sinne u.a. Kollokationen, kommunikative Phraseme und Sprichwörter und zu den Phrasemen im engeren Sinne die Idiome. Phraseme werden nach den Funktionen, die sie in der Kommunikation erfüllen, in referentielle, strukturelle und kommunikative Phraseme unterteilt. Referentielle Phraseme benennen und beschreiben Objekte, Vorgänge oder Sachverhalte, strukturelle Phraseme stellen syntaktische Beziehungen her und kommunikative Phraseme übernehmen bestimmte Aufgaben „,bei der Herstellung, Definition, dem Vollzug und der Beendigung kommunikativer Handlungen.“ (Ebd.: 31)

\section{3}

\section{ZUM FORSCHUNGSSTAND}

Die Phraseologieforschung, die sich seit ihren Anfängen in den 70er und 80er Jahren des 20. Jahrhunderts mit der Definition und Klassifikation von Phrasemen auseinandersetzt, widmete sich später den komplexen Fragen des phraseologischen Gebrauchs in verschiedenen Textsorten. Der systemhafte Untersuchungsaspekt in den phraseologischen Studien wird mit dem Begriff „textbildende Potenzen” von Černyševa (zit. n. Dobrovol'skij 1980: 690) um den textbezogenen bzw. pragmatischen Aspekt erweitert. Es geht um die spezielle Fähigkeit von Phrasemen, die aufgrund ihrer besonderen Beschaffenheit bestimmte Funktionen im Text übernehmen und an der Konstruktion der Textstruktur mitwirken können. Kühn (1985) hebt den phraseologischen Mehrwert von Phrasemen, ihre expressiven, wertenden, emotionalen, handlungsanweisenden u.a. Komponenten in der textuellen Verwendung hervor. In vielen Studien werden Beobachtungen zum Vorkommen von Phrasemen, ihre Erscheinungsformen und Verwendungsweisen in Texten untersucht (vgl. Burger 1997, Richter-Vapaatalo 2007, Mellado Blanco 2008, Lenk/Stein 2011, Pavić-Pintarić 2015, Pfeiffer 2016, Jesenšek 2021). ${ }^{1}$ Auch Phraseme in der Werbung sind Gegenstand zahlreicher Forschungsarbeiten. Entweder konzentrieren sich die Studien hierbei auf die Werbesprache, in der Phraseme eine Perspektive unter vielen anderen darstellen (vgl. Fritz 1994, Janich 2001, Stöckl 2004) oder auf Phraseologie in der Werbesprache, die als Hauptgegenstand der Analyse erläuert wird (vgl. Grasseger 1989, Hemmi 1994, Lange 1998, Balsliemke 1999, Balsliemke 2001, Bass 2003, Janich 2005, Janich 2006, Vesalainen 2007, Ptashnyk 2009, Majnusz-Stadnik 2014, Rypáčková 2019, Umborg 2020). In Werbeanzeigen dienen Phraseme der Persuasion, weil sie dazu

1 Eine Übersicht über die Forschung geben Sabban (2007) und Valenčič Arh (2014: 80). 
beitragen können, dass die Botschaft einprägsam und konnotationsreich klingt, manchmal auch unterhaltsam, wenn Phraseme sprachspielerisch eingesetzt werden (vgl. Bachmann-Stein 2011: 35) In der Untersuchung von Grasseger (1989) werden Phraseme im engeren Sinne in 400 Werbespots in der österreichischen Fernsehwerbung analysiert. Nach seinen Ergebnissen kommen Phraseme darin sehr selten vor, nur in 5 Prozent. Auch Burger (1991) geht der Frage nach der Rolle der Phraseme in der Fernsehwerbung nach und stellt fest, dass Phraseme in der Hälfte von den 230 von ihm analysierten Spots vorkommen. In seiner Studie, in der Lange (1998) die Verwendung sprachlicher Vorlagen (d.h. Phraseme und Sprichwörter) in Texten der Anzeigenwerbung unter Berücksichtigung der Intentionalität der Werbung untersucht, wird auf unterschiedliche Funktionen hingewiesen, vor allem auf die Aufmerksamkeitssteigerung beim Leser. Der Verwendung von Phrasemen lassen sich positive Aspekte abgewinnen, weil Werbetexter beim Umgang mit der Sprache Kreativität beweisen (vgl. Lange 1998: 195). Hemmi (1994) untersuchte die Häufigkeit, die Modifikationsverfahren und Positionierung der Phraseme in der schweizerischen Anzeigen-, Radio- und Fernsehwerbung und ihre Rezeption mittels einer Umfrage unter erwachsenen Versuchspersonen. Es stellte sich der partiell unterschiedliche Gebrauch von Phrasemen in den drei Medien heraus, der auf die Möglichkeiten der jeweiligen Einsetzung von visuellen und akustischen Darstellungsmöglichkeiten zurückzuführen ist. Balsliemke (2001) konzentrierte sich auf das Vorkommen, die phraseologischen Typen und Modifikationen der Phraseme sowie auf ihre Text-BildKohärenz und Vertextung in der Anzeigenwerbung. In ihrer diachronen Untersuchung der Phraseologie in der Deutschschweizer Anzeigenwerbung von 1928 bis 1998 stellte Bass (2003: 383) fest, dass Phraseme bei den Werbetextern bereits in der ersten Hälfte des 20. Jahrhunderts als attraktives und vielseitiges Gestaltungsmittel beliebt waren. Janich (2005) hebt die auffällige und unauffällige Verwendung von Phrasemen in verschiedenen Teilen der Anzeigenwerbung, d.h. in Schlagzeilen oder Slogans, hervor. Nach dem Ergebnis ihrer Untersuchung dienen Phraseme in der Werbung einerseits der kognitiven Entlastung und dem leichten Verständnis der Botschaft und andererseits erzielen sie stilistische Effekte wie Mehrdeutigkeit, Humor und Anschaulichkeit.

\section{4}

\section{AUFBAU KOMMERZIELLER WERBEANZEIGEN}

Laut Janich (2001: 43ff.) besteht eine kommerzielle Werbeanzeige aus folgenden Bestandteilen: der Schlagzeile, dem Fließtext, dem Slogan, dem Visual, besonderen Textelementen wie z.B. dem Bestellcoupon und dem Logo (vgl. Balsliemke 2011: 15ff.; Fritz 1994: 157). Die Schlagzeile (auch Headline oder Aufhänger) als zentrales Textelement und wichtiger Blickfang bietet einen Leseanreiz für die weitere Beschäftigung mit der Anzeige. Mit dem Slogan werden die im Bewusstsein der Käufer hervorgerufenen Assoziationen verknüpft, die das Produkt und das werbende Unternehmen miteinander 
verbinden. Im Fließtext wird die Werbung thematisch beschrieben, der Aufhänger aus der Schlagzeile in einer kohärenten Sprachform beschrieben, so dass der potenzielle Käufer detaillierte Informationen zur Verwendung des Produktes finden kann. Das Visual stellt die nonverbale Komponente dar und erstreckt sich von Objekten, Situationen oder Personen bis zu Symbolen. Janich (2001: 60) versteht die Bildelemente als solche Teile der Werbung, die zuerst wahrgenommen und inhaltlich schneller als der Text erfasst werden, weil sie ,assoziationsreich sind und emotionale Inhalte vermitteln“ können. Einige besondere Textelemente gehen über werbende Informationen zum Produkt hinaus, wie z.B. das Logo, der Bestellcoupon, gesetzlich vorgeschriebene Textbausteine usw. Das Logo ist eine stilisierte graphische Darstellung des Waren- oder Firmenzeichens. Manche Werbeanzeigen enthalten auch einen Bestellcoupon, der der zweiseitigen Kommunikation mit dem potenziellen Konsumenten dient. Man kann ihn abschneiden und an die Firma schicken. Diese informiert den Käufer über weitere Details eines Produktes. $\mathrm{Zu}$ den gesetzlich vorgeschriebenen Textbausteinen gehört z.B. bei der Zigarettenwerbung die Warnung (Rauchen gefährdet die Gesundheit).

\section{$5 \quad$ AUFFÄLLIGE UND UNAUFFÄLLIGE VERWENDUNG DER PHRASEME}

Wenn Phraseme in modifizierter Form an die jeweiligen kommerziellen Interessen angepasst werden, tragen die Modifikationen zusätzlich zur Aufmerksamkeitssteigerung bei. Was sind aber eigentlich Modifikationen? Das Merkmal Festigkeit (auch Stabilität genannt) und die damit einhergehende Reproduzierbarkeit besagen, dass Phraseme wie einzelne Lexeme gebräuchlich sind und den Sprechern als formelhafte Ausdrücke zur Verfügung stehen, denn sie existieren als vorgefertigte sprachliche Einheiten in einer Sprachgemeinschaft und reflektieren zugleich deren Wahrnehmungs- und Erfahrungswelt. Hegedüs-Lambert (2010) erklärt, „dass ein bestimmter kognitiver Inhalt sich im Phrasemsystem festigen kann und durch das Phrasemsystem zugänglich wird." Bei der Sprachproduktion bieten Phraseme als feste formelhafte Formen die kognitive Entlastung und in ritualisierten Situationen auch Verhaltenssicherheit.

Phraseme werden in Texten in unveränderter oder in veränderter Form verwendet. Wenn Phraseme als „bloßer Baustein” des Textes unauffällig verwendet werden, heißt das laut Sabban (2004: 239), dass mit ihrer Einbindung in den Kontext kein besonderer Mehrwert stimuliert wird. Phraseme halten sich morphosyntaktisch an bestimmte Regeln der Syntax, semantisch behalten sie ihre phraseologische Bedeutung. Unauffällig verwendet werden Phraseme, wenn sie in ihrer phraseologischen Bedeutung gebraucht werden und für den betreffenden thematischen Kontext typisch sind.

Wegen ihrer komplexen Mehrwortstruktur, der relativen Festigkeit, der okkasionellen Modifikation und der metaphorischen Prozesse, die der Idiomatizität zugrunde liegen, 
werden Phraseme in Texten oft modifiziert, d.h. auffällig verwendet. Diese kreative Verwendung entfalten Phraseme als sekundäre sprachliche Zeichen, die im Unterschied zu Einzellexemen zu den verschiedenen Textdimensionen einen besonderen Beitrag leisten können (vgl. Sabban 2007: 237). Modifizierte Phraseme regen den Rezipienten zum Nachdenken an, wobei er das modifizierte Phrasem mit seiner lexikalisierten Nennform vergleichen kann. Im Gegensatz zu den usuellen Bildungen sind Modifikationen solche okkasionellen Erscheinungen, die für einen bestimmten Text abgewandelt werden (vgl. Burger 2015: 27, Fleischer 1997: 205). Stein (1995: 116ff.) sieht gerade in der Formelhaftigkeit die Grundlage für verschiedenartige Neubildungen und Veränderungen der festen Wortverbindungen. Erst die Musterhaftigkeit eröffnet die Möglichkeit für kreative Innovation, okkasionelle intertextuelle Anspielungen, für die auffällige Verletzung der festen Regeln und die Auflösung der starren Formelhaftigkeit. Indem man die Regeln bricht, bestätigt man ihre Existenz. Laut Fix (1997: 104f.) zieht man aus dieser Verletzung der Regeln einen stilistischen Gewinn. Ptashnyk (2009: 55) definiert phraseologische Modifikationen als ,alle okkasionellen Transformationen der Struktur und/ oder Semantik der PE in der Rede, die nicht für die morphosyntaktische Einbettung des Phraseologismus im Satz notwendig sind." Es werden folgende pragmatische Funktionen phraseologischer Modifikationen differenziert: 1. Leseranreiz/Motivation des Lesers, 2. Aufmerksamkeitssteuerung, 3. Beeinflussung des Lesers, 4. Unterhaltung des Lesers, 5. Hervorrufen metasprachlicher Reflexionen beim Leser (vgl. ebd.: 224 ff.).

Nach Burger (2015: 162 ff.) werden Modifikationen in einem konkreten Text gebraucht und sind deshalb auch nur im Kontext verständlich. Dabei unterscheidet Burger (ebd.) zwei Arten von Modifikationen. Bei lexikalisch-morphosyntaktischen (oder formalen) Modifikationen wird in die äußere Form und Struktur des Phrasems eingegriffen. ${ }^{2}$ Bei semantischen Modifikationen ist der Fokus auf die Bedeutung des Phrasems oder der einzelnen Komponenten gerichtet. In der vorliegenden Untersuchung stellen die semantischen Modifikationen die Grundlage für die Analyse der Text-Bild-Beziehungen dar.

In den Anzeigetexten wird eine schnelle Rezeption auch durch die visuelle Kommunikation mittels Bild-Elementen erreicht. Die nonverbalen Informationen liefern zusätzliche Bilder im Visual. Die Verknüpfung der verbalen und nonverbalen Komponenten der gesamten Werbeanzeige wird als Text-Bild-Beziehung bzw. Text-Bild-Bezug bezeichnet

$2 \mathrm{Zu}$ den häufigsten formalen Modifikationen im Textteil des verwendeten Anzeigenkorpus zählen (1) die Substitution, wenn einzelne Komponenten ausgetauscht werden (,Wissen ist Geld“ statt Zeit ist Geld), (2) die Ergänzung, wenn dem lexikalisierten Phrasem weitere Komponenten hinzugefügt werden (,So viel Wind kann ein Diesel machen" aus (viel) Wind (um etwas) machen) und (3) die Verkürzung oder Ellipse, wenn einzelne Komponenten ausgelassen werden (,Mit Cabrio ins Blaue“ aus eine Fahrt ins Blaue machen). 
(vgl. Balsliemke 2001: 30). ${ }^{3}$ Auf diese Weise kommt es zu semantischen Modifikationen, die der spezifischen, kreativen und spielerischen Einbettung der Phraseme in den Kontext dienen. Das Bild kann eine Beziehung zur Werbebotschaft oder zur Darstellung des Nutzens, der durch den Kauf des Produktes bewirkt wird, oder auch zu dem im Text verwendeten Phrasem bzw. zu einer seiner Komponenten ausdrücken. Sobald in dieser Beziehung die wörtliche Lesart des Phrasems zusätzlich zur phraseologischen Lesart aktualisiert wird, spricht man von Modifikation in semantischer Hinsicht. Genauer gesagt werden auf der Textebene beide Lesarten aktualisiert. Das Grundprinzip der semantischen Modifikationen basiert auf dem Wechselspiel der phraseologischen und der wörtlichen Lesart. Dadurch kommt es zur Doppeldeutigkeit (o. Mehrdeutigkeit) bzw. zum semantischen Effekt, den man Ambiguierung nennt. ${ }^{4}$ Ausschlaggebend ist dabei immer der Kontext.

Jeder phraseologische Ausdruck kann innerhalb eines Gesamttextes einen spezifischen Bezug zum materiellen Bild herstellen. Umgekehrt können auch materielle Bilder auf die Lesarten der Phraseme referieren, ohne direkt visualisiert zu werden. Sie können nur einzelne Hinweise in Form der phraseologischen Komponente, als entsprechende Handlungskontexte oder assoziierbare Konzepte u.a. sein. Laut Stöckl (2004: 327) ist prinzipiell zwischen ,der mehr oder weniger direkten Visualisierbarkeit eines phraseologischen Ausdrucks bzw. seiner Teile und der indirekten und sehr variablen semantischen Verknüpfbarkeit des Bildes mit dem Ausdruck" zu unterscheiden. Wenn Phraseme über eine sensorisch-anschauliche Komponente verfügen, können sie direkt oder indirekt auf ein materielles Bild bezogen werden (vgl. ebd: 310). Wie intensiv der Bildbezug ist, hängt von der Intensität/Art des semantischen Zusammenhanges zwischen den Bedeutungen der Phraseme (oder deren einzelnen Komponenten) und den Bedeutungen der bildlichen Darstellung (oder deren visuellen Elementen) ab.

Die vorliegende empirische Analyse der Phraseologie in der Anzeigenwerbung beruht auf der Typologie von Stöckl (2004: 311), der zwei Typen (mit weiteren Ausprägungen) des Bildbezuges von Phrasemen im Text unterscheidet: den latenten und den expliziten Phrasem-Bild-Bezug.

Beim latenten Phrasem-Bild-Bezug (Typ I) wird das entsprechende Phrasem nicht materialisiert, jedoch besteht ein semantischer Bezug zwischen sprachlichem Bild und materiellem Bild. Von visuell evoziertem Phrasem ist die Rede, wenn das Bild ohne

3 Im Rahmen der Text-Bild-Beziehung unterscheidet Balsliemke (1999: 25) zwei Begriffe: Text-Bild-Kohärenz und Vernetzung. Wenn sich der Text der Überschrift und das Bild auf den gleichen Sachverhalt beziehen, dann geht es um Text-Bild-Kohärenz. Bei der Vernetzung, werden ,die verbalen und visuellen Komponenten einer Anzeige nach einer Text-Bild-Beziehung weitergehend durch vielfältige Anspielungen miteinander"verknüpft, wie z.B. synonyme oder antonyme Relationen zw. dem Phrasem und anderen Lexemen in der Werbeanzeige.

4 Im Handbuch der Phraseologie wird Ambiguierung u.a. definiert als Oberbegriff für weitere Formen der Doppeldeutigkeit und „(...) bezieht sich auf Phraseologismen mit einer (oder mehreren) potentiellen wörtlichen Bedeutung(en). Bewußtes Aktualisieren beider (allenfalls mehrerer) Bedeutungsebenen, ohne Rücksicht darauf, welche der Ebenen im Kontext als dominant gemeint ist. (Neutraler) Oberbegriff für Literalisierung, Remotivierung, WörtlichNehmen und Polysemantisierung.“ (Burger/Buhofer/Sialm 1982: 29) 


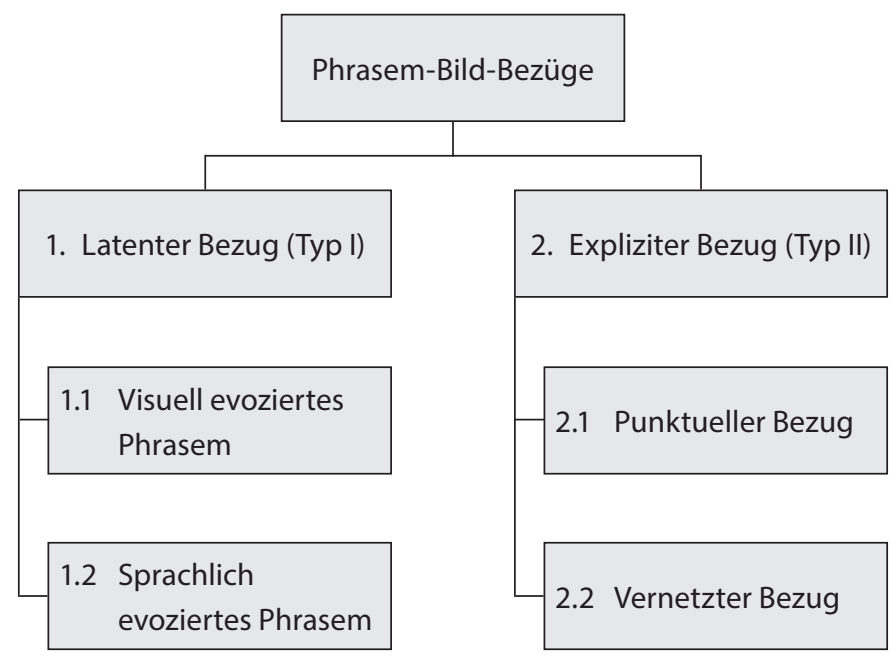

Abb. 1: Typen von Phrasem-Bild-Bezügen nach Stöckl (2004: 311)

Sprache auf ein Phrasem, das im Text nicht explizit erscheint, hindeutet. Dagegen sind mit sprachlich evoziertem Phrasem solche semantischen Gebilde gemeint, bei denen die Sprache in unterschiedlich starkem Maße an der Erweckung von Vorstellungen über ein Phrasem beteiligt ist und über die phraseologische Lesart ergänzend auf die Werbebotschaft hinweist.

Der explizite Phrasem-Bild-Bezug (Typ II) bezeichnet solche Konstellationen, in denen Phraseme explizit in Bild und Text materialisiert werden. Wenn das Phrasem auffällig nur an einer markanten Stelle, entweder in der Schlagzeile, im Slogan oder im Fließtext positioniert ist, dann geht es um den ,punktuellen Bezug“ (2.1). Wenn aber das Phrasem auf auffällige Art und Weise durch andere Textelemente an anderen Textstellen gestützt wird, dann wird diese Konstellation als „vernetzter Bezug“ (2.2) bezeichnet.

\section{7}

\section{BEISPIELANALYSE}

Die in diesem Beitrag analysierten Beispiele sind Teil eines Korpus, das aus 84 Werbeanzeigen besteht, die zwischen 2006 und 2021 in verschiedenen deutschsprachigen Zeitschriften und Magazinen publiziert wurden. Nach dem Zusammenstellen des Korpus wurden die belegten Phraseme in eine Excel-Tabelle eingetragen. Zuerst wurde zu jedem Phrasem die lexikalisierte Bedeutungsparaphrase aus dem Wörterbuch DUDEN 11 angeführt. Als zusätzliche Hilfe wurden die Phraseme auf der Internetseite redensarten-index.de nachgeprüft. Sofern die Phraseme in modifizierter Form auftraten, wurde auch dies in die Tabelle eingetragen. In der nächsten Phase wurden alle Belege je nach Kontext den einzelnen Phrasem-Bild-Bezügen (vgl. Abb. 1) zugeordnet. Es ging bei der Analyse nicht 
um die isolierten Funktionen der Phraseme, sondern um zu untersuchen, welche Beziehungen zwischen Text und Bild mittels Phrasemen hergestellt werden können. Aus Platzgründen wird in der Beispielanalyse zu jedem Phrasem-Bild-Bezug nur ein Beleg ausgewählt. Nur beim punktuellen Bezug werden zwei Werbeanzeigen gezeigt, weil in dieser Gruppe des Text-Bild-Bezuges zwei Ausprägungen unterschieden werden können: mit formal modifizierten Phrasemen oder nicht modifizierten Phrasemen.

\subsection{Typ I: Visuell evozierte Phraseme}

Wenn Phraseme ohne entsprechende materielle Bilder verwendet werden, sind phraseologische Formulierungen indirekt assoziativ, d.h. der Wissensrahmen wird nicht visualisiert, sondern assoziativ evoziert. Im Bildbezug werden zwar mögliche wörtliche Lesarten aktiviert, aber nicht direkt abgebildet. Stöckl (2004: 317) bezeichnet solche Konstruktionen als unauffällig, ,weil die sprachlich generierte Bildlichkeit hier durch visuelle Elemente nicht fokussiert wird.“

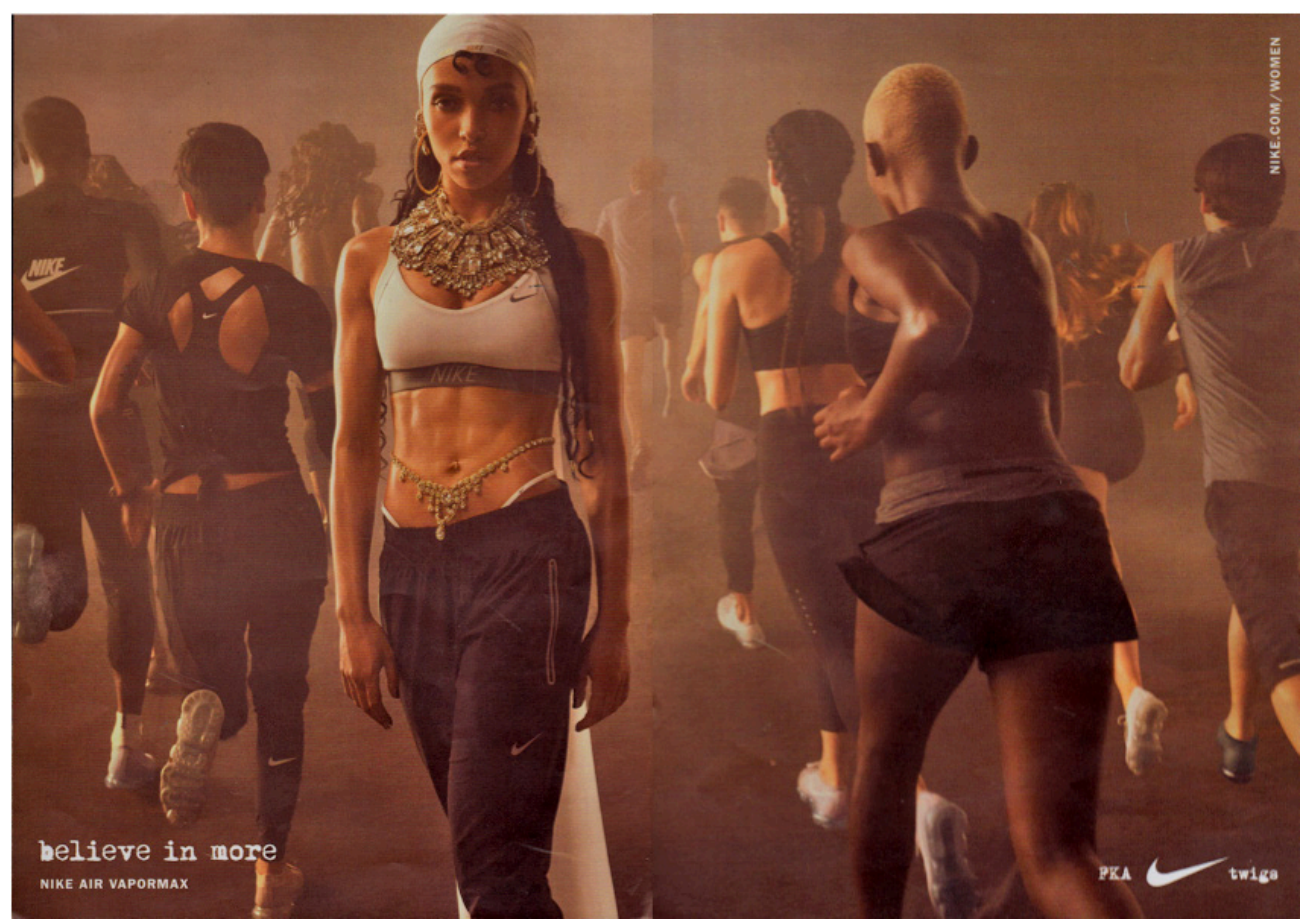

Abb. 2: Werbeanzeige der Firma Nike (2017)

In der Gruppe der visuell evozierten Phraseme werden solche semantischen Gebilde zusammengefasst, bei denen das Bild auf ein Phrasem hindeutet, das im Text aber nicht 
verbalisiert wird. Für das Verstehen der Anzeige ist das Erkennen der impliziten Hinweise, die auf das nichtvisualisierte Phrasem hindeuten und suggestive Vorstellungen erwecken, von entscheidender Bedeutung.

In unserem Korpus konnten zwei Belege (2\%) mit visuell evoziertem Phrasem festgestellt werden, von denen einer im Folgenden beschrieben werden soll. Da aus Platzgründen nicht auf alle analysierten Werbeanzeigen eingegangen werden kann, wurde die Anzeige der Firma Nike ausgewählt. Auf dieser Anzeige in der Abbildung 2 sieht man Läufer, die dem Betrachter den Rücken zuwenden. Nur eine Frau in einem attraktiven sportlichen Outfit bewegt sich in langsamem Tempo und mit energischer Körperhaltung und einem zielsicheren Blick auf das Objektiv hin. Die bildliche Darstellung deutet auf das Phrasem jemandem / einer Sache den Rücken kehren hin, obwohl die Beziehung zum Phrasem an keiner Stelle explizit angeführt wird. Die bildliche Darstellung evoziert das Phrasem in seiner Bedeutung: Die Frau, die nicht nur Sportkleidung der Marke Nike, sondern auch Halsschmuck, Ohrringe, eine Bauchkette sowie ein Kopftuch trägt, kehrt den anderen den Rücken zu. Die Kenntnis des Phrasems ist ausschlaggebend für das Verständnis der Bildidee. Bei näherer Betrachtung der gesamten Anzeige wird das Phrasem aktualisiert und seine phraseologische Lesart 'jemanden / etwas verlassen; sich abwenden; etwas aufgeben' kommt zum Tragen. Der Slogan believe in more links unten auf dem Bild signalisiert, dass sich die Frau mit ihrem aufwändigen Outfit von den anderen Sportlern abwendet und eine gewisse Distanz schafft.

\subsection{Typ I: Sprachlich evoziertes Bild}

Phraseme werden in Werbeanzeigen auch unauffällig ohne entsprechende materielle Bilder gebraucht. Mentale Bilder können durch Phraseme aufgebaut werden, stellen aber keine direkte Materialisierung in einer bildlichen Darstellung dar. Die fehlenden Pole des Bezugs werden aber aktiviert. Das Phrasem deutet über seine wörtliche Lesart potenziell auf die Visualisierung hin, die im Text mitschwingt, wird aber bildlich nicht konkret dargestellt. Für den aktuellen Werbetext ist nur die phraseologische Lesart dominant.

In dieser Gruppe konnten 13 Belege (16\%) mit sprachlich evoziertem Bild gefunden werden. In der Werbeanzeige der Firma Telekom Austria wird ein fröhlicher Musketier im modernen Gewand gezeigt, der seine Begeisterung über eine einwandfrei funktionierende Internetverbindung auf dem Computer mit einer typischen HurraGeste zum Ausdruck bringt. Über der Figur steht die fettgedruckte und große Schlagzeile „A1 Breitband für zuhause. Einfach für alle.“, die neben dem Bild das zentrale Textelement darstellt. Seine Figur befindet sich im Mittelpunkt der Abbildung und nimmt zwei Drittel der gesamten Fläche der Werbeanzeige ein. Es sieht so aus, als 
ob er in der Luft schweben würde. Seine rechte Hand ist auf die Tastatur gerichtet, als wollte sie gleich mit dem Tippen beginnen. Die linke Hand streckt er in die Luft und macht dabei eine Faust. Er hält sich aufrecht, das linke Bein ist nach vorne ausgestreckt. Es hat den Anschein, als wollte er seinen linken Fuß in diesem Moment nach vorne setzen, während sein rechtes Bein nach hinten gebeugt ist. Sein Gewand ähnelt ebenso dem Kostüm von Superman. Dieser ist mit übernatürlichen Kräften ausgestattet und besitzt eine High-Tech-Ausrüstung. Die Wiedererkennung aufgrund des typischen Kostüms erweckt eine positive Einstellung zum Produkt, denn Superman steht für den Mut und die Macht, über die jeder Mensch verfügt. Links unter dem Bild steht der Fließtext: „Ob Laptop, PC oder Tablet: Zuhause ist es doch am schnellsten. Unlimitiertes, ungebremstes A1 Breitband-Internet mit WLAN für zuhause - inkl. kostenloser Herstellung. Und dazu A1 TV jetzt 6 Monate gratis. Einfach so." Rechts neben dem Text folgen als bildhafte Reproduktion ikonische Zeichen für Computer und Fernsehen, die zur Verdeutlichung visualisieren. Darunter steht der Preis. Ganz rechts wird der Slogan „Einfach A1“ wiederholt. Das bereits durch die Figur des Musketiers visuell evozierte Phrasem Einer für alle wird sprachlich assoziiert und zugleich festgelegt. Das lateinische Zitat unus pro multis stammt aus Vergils Äneis. Es steht für Zusammengehörigkeit, für Empathie und Füreinander einstehen in einer Gruppe (vgl. DUDEN 2007: 210). In der Erweiterung Einer für alle, alle für einen steht es als Motto im Roman Die drei Musketiere von Alexandre Dumas. Hier ist die Sprache an der Evokation des Phrasems beteiligt. Solche Text-Bild-Kombinationen erwecken die Aufmerksamkeit sicherlich aufgrund der zunächst nicht eindeutigen Konzeptassoziation. Erst der Bezug auf die sprachliche Textumgebung macht die zentrale Aussage deutlich und erleichtert die Verständlichkeit.

\subsection{Typ II: Punktueller Phrasem-Bild-Bezug}

Beim expliziten Phrasem-Bild-Bezug werden Phraseme im Text und auf dem Bild auf unterschiedliche Weise explizit aktualisiert. Auf dem Bild können Phraseme oder einzelne Komponenten in wörtlicher Lesart dargestellt werden oder es wird ein Bezug zum Produkt bzw. zur nachfolgenden Situation, wenn das Produkt in der Tat gekauft wird, hergestellt. Der ganze Text steuert die Deutung von beiden Seiten (Text und Bild).

Nach den Ergebnissen der Analyse entfallen auf den punktuellen Phrasem-BildBezug 31 Belege (37\%). Zu dieser Gruppe werden solche Gebilde gezählt, bei denen ein auffälliges Phrasem nur an einer herausragenden Stelle, d.h. punktuell eingesetzt wird. Wenn das Bild im Visual einen direkten Bezug zum Phrasem in der Schlagzeile oder im Fließtext herstellt, wird dieser Bezug in Sprache und Bild zugleich parallel expliziert. Das Phrasem (oder eine seiner Komponenten) wird mit dem materiellen Bild 
verbunden. Die bildliche Darstellung öffnet einen semantischen Wissensrahmen zum Thema, das alle abgebildeten Elemente miteinander verknüpft. Da bei dieser Gruppe des Text-Bild-Bezuges zwei Ausprägungen unterschieden werden können, je nachdem, ob Phraseme formal modifiziert werden oder nicht, werden im weiteren Text zwei Werbeanzeigen beschrieben.

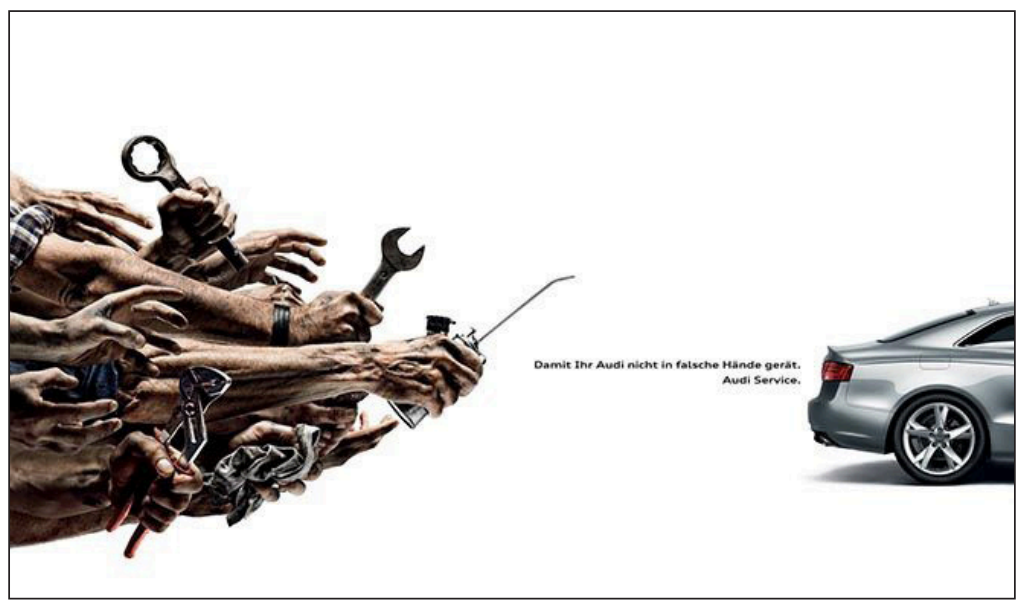

Abb. 3: Werbeanzeige für Audi Service (2015)

Auf der Anzeige für Audi Service (Abbildung 3) aktiviert das materielle Bild zugleich eine wörtliche und eine phraseologische Lesart. Die Werbung betextet die bildliche Darstellung auf der linken Seite, auf der eine Vielzahl von Händen verschiedene Werkzeuge halten, mit dem Text: „Damit Ihr Audi nicht in falsche Hände gerät. Audi Service." In falsche Hände geraten bedeutet im phraseologischen Sinne 'von jemandem in Besitz genommen werden, der damit nicht umgehen kann /, für den es nicht vorgesehen war /, der damit etwas Schädliches tut'. Die visuell präsentierte Szene der schmutzigen Hände der Mechaniker ist dem Betrachter aus dem Alltag bekannt und ruft automatisch eine Erfahrung beim Autoservice wach. Auf dem Bild, das auf der wörtlichen Bedeutung beruht, wird die möglich falsche Servicenutzung/-leistung illustriert und es appelliert zugleich mit der Negation ,nicht“ an den Betrachter, die eventuellen Folgen in Betracht zu ziehen. Die Bildkomposition leistet zweierlei: Einerseits wird die phraseologische Lesart gestützt und andererseits demonstriert das Bild zugleich zwei Bedeutungsebenen des Phrasems. Aufgrund des Bildbezugs wird das Verfahren der Remotivierung eingesetzt, d.h. die wörtliche Lesart wird aktualisiert. Sabban (2007: 241) spricht von „Ambiguierung durch Evozieren der Wörtlichkeit“. Die pragmatische Gesamtbedeutung liegt zwischen der kognitiven Entlastung und dem schnellen Verständnis durch die Anknüpfung an Bekanntes und dem sprachspielerischem Effekt. 


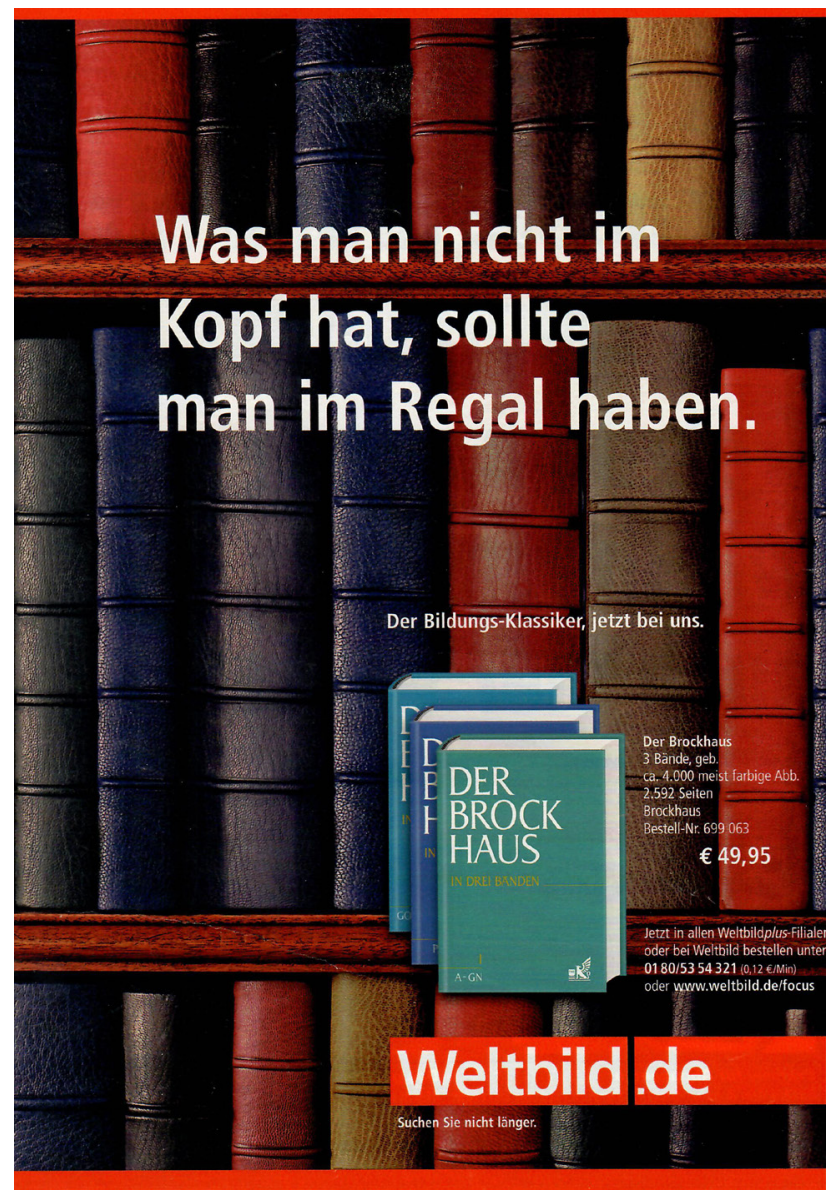

Abb. 4: Anzeige der Verlagsgruppe Weltbild für das Lexikon Brockhaus (2004)

In der Anzeigenwerbung auf der Abbildung 4 wird das Sprichwort Was man nicht im Kopf hat, hat man in den Füßen / Beinen in der Bedeutung 'Wer sich nichts merken kann, muss oft noch einmal umdrehen, weil er was vergessen hat' dem Produkt angemessen modifiziert. Da dieses Sprichwort die Komponente Kopf enthält, wird es zu den Somatismen gezählt, denn Somatismen stellen die Gruppe von Phrasemen dar, die einen Körperteil als Komponente haben (griech. soma = Körper). Laut Bánffi-Benedek (2016: 250) liegen den Somatismen ,universelle, aber auch kulturspezifische, oder kulturspezifisch metaphorische und emotionale Konzepte zugrunde“. Dobrovol'skij (1997: 126f.) sieht Körperteilbezeichnungen in Phrasemen als Quasisymbole und Kopf steht hier als 'Ort bzw. Instrument des Denkens, der intellektuellen Tätigkeit'. Das Visual stellt ein Buchregal dar. Die durch das Verfahren der lexikalischen Substitution formal modifizierten Komponenten in den Beinen werden durch die Lexeme im Regal ersetzt. Dadurch 
entsteht eine Beziehung zwischen dem textuellen und visuellen Teil der Anzeigenwerbung. Die Aktualisierung der wörtlichen Lesart entsteht durch den Bild-Bezug. Es entstehen metakommunikative Bezüge zwischen dem Phrasem und dem Bild. Die Montage der bildlichen Darstellung rückt das Phrasem in den Fokus der Aufmerksamkeit.

\subsection{Typ II: Vernetzter Phrasem-Bild-Bezug}

Nach unserer Analyse stellt der vernetzte Phrasem-Bild-Bezug mit 38 Belegen (45\%) die größte Gruppe dar. Im nächsten Beispiel wird eine Text-Bild-Konstellation gezeigt, in der mehrere Phraseme verwendet werden und miteinander semantisch vernetzt sind. Bei einem vernetzten Phrasem-Bild-Bezug geht es darum, dass die phraseologischen Lesarten die Sprachhandlungsstruktur des Werbetextes systematisch bestimmen.

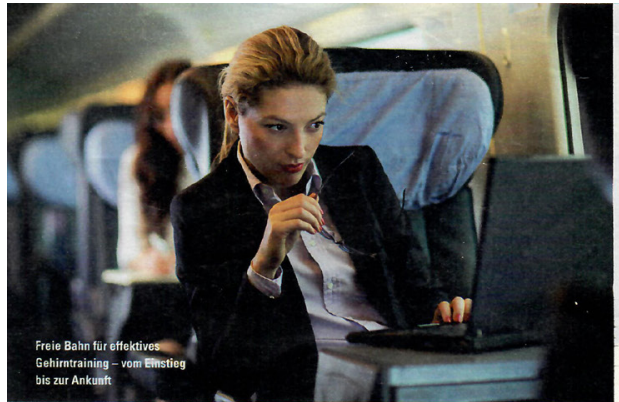

Hier kommen Ihre grauen Zellen in Fahrt

Konzentrations-Steigerung leicht gemacht: Bahnfahrer können die Zeit im Zug für wirkungsvolle Übungen zur Verbesserung der geistigen Leistungsfähigkeit nutzen

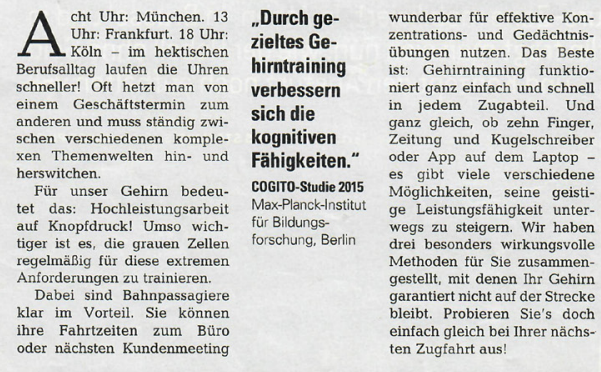

Diese Zeit gehört Dir.

Am besten lessen Sie sich gleich auf dem Online-Portal der Deutschen Bahn hahn.de/deinezeit inspirieren, wie Sie lhre Zeit im Zug über wirkungsvolles Konzentrations - und Gecàchtnistraining hinaus effektiv gestalten können. Probieren Sie bei lhrer nächsten Bahnfahrt einfach mal etwas Neues aus! Machen Sie ein Foto oder Video davon und laden Sie es auf der Website hoch. Mit etwas Glück gewinnen Sie eine Städtereise von Amerope oder einen der attraktiven Wachenpreise.

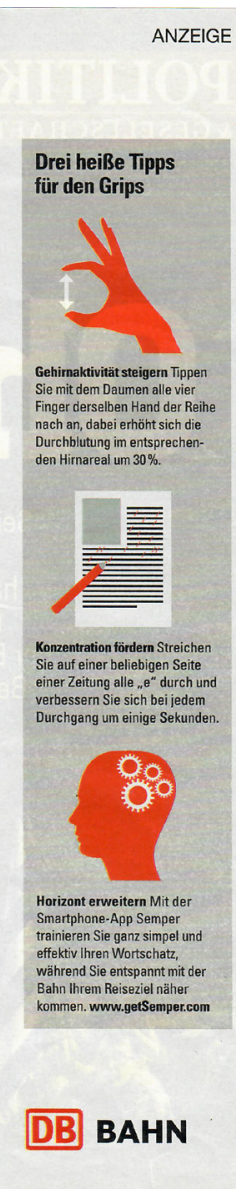

Abb. 5: Werbeanzeige für Deutsche Bahn (2015) 
In der Werbeanzeige für die Deutsche Bahn auf der Abbildung 5 werden zwei Phraseme zusammengeführt: graue Zellen und in Fahrt kommen. Mit dem nominalen Phrasem graue Zellen ist die Denkfähigkeit bzw. geistige Leistungsfähigkeit gemeint. Beim Phrasem in Fahrt kommen handelt es sich auf der konkreten Ebene um Bewegung und den Vorgang des Fahrens und auf der zweiten, metaphorischen Ebene um die Bedeutung 'in Schwung, in gute Stimmung kommen' (DUDEN 2002: 206).

In semantischer Hinsicht steuert der Kontext die jeweilige Lesart und primär ist das Phrasem im phraseologischen Sinn gemeint. Aus den Informationen im Fließtext ist ersichtlich, dass man mit dieser Anzeige daraufhin aufmerksam machen will, dass bei einer Zugfahrt die Zeit zur Verbesserung der Leistungsfähigkeit genutzt werden kann. Alle anderen Phraseme und bildhafte Wendungen sind indirekt assoziativ: freie Bahn für effektives Gehirntraining (...), (...) Hochleistungsarbeit auf Knopfdruck; (...) nicht auf der Strecke bleiben, (...) laufen die Uhren schneller usw. Die Häufung der phraseologischen Sprache aktiviert ein lexikalisch-semantisches Beziehungsnetz, das die möglichen Konzepte einer Zugfahrt eröffnet und beim Betrachter hervorruft. Laut Burger (2015: 166) werden in solchen Text-Bild-Konstruktionen Phraseme mit potentiell zwei Lesarten semantisch durch den Kontext gesteuert. Je nach Aktualisierung der einzelnen Lesart gibt es verschiedene Möglichkeiten. Es kann die phraseologische Lesart oder die wörtliche dominieren, beide zugleich oder es entsteht eine neue Lesart (ebd.).

\section{8}

\section{SCHLUSSFOLGERUNG}

In diesem Beitrag wurde die Phraseologie in Werbeanzeigen der Printmedien analysiert. Es wurden Werbeanzeigen, in denen Phraseme im Bild und/oder im Text verwendet werden, in Betracht gezogen. Das Ziel der Analyse war es zu zeigen, welchen Beitrag Phraseme in den Werbeanzeigen sowohl im Bild als auch im Text leisten können. Im Vordergrund standen die Fragen, ob Phraseme eine latente oder eine explizite Beziehung zum Bild und/oder Text herstellen und welche semantischen Leistungen Phraseme für die Wahrnehmung und das Verständnis des gesamten Textes erbringen.

Die durchgeführte Analyse von Phrasemen in den Werbeanzeigen zeigt, dass Phraseme gezielt sowohl im Text als auch im Visual eingesetzt werden. Es konnte festgestellt werden, dass es sehr wenige latente Phrasem-Bild-Bezüge gibt, bei denen keine direkten materiellen Abbildungen der Phraseme gezeigt werden. In nur 18 Prozent der Belege gibt es zwischen sprachlichem und materiellem Bild nicht unmittelbar sichtbare Beziehungen. Dagegen dominieren in 82 Prozent der untersuchten Werbeanzeigen solche Konstellationen, in denen Phraseme auffällig und sichtbar im Bild und im Text eingesetzt werden. Daneben werden sie oft auch durch andere Textelemente vernetzt.

Es kann bestätigt werden, dass man mit der Werbesprache durch ein komplexes Zusammenwirken von Text- und Bildelementen versucht, die Aufmerksamkeit der 
Konsumenten auf ein bestimmtes Produkt zu lenken und sie anschließend zum Kauf dieses Produkts anzuregen. Durch Sprache, attraktive Gestaltung und Bild wollen Werbetexter die Kunden für sich gewinnen. Die Aufmerksamkeit wird über ein komplexes Zusammenwirken von Text- und Bildelementen gelenkt. Das Bild und die Sprache werden aufeinander abgestimmt: Der Text übernimmt die stabilisierende oder erklärende Funktion, das Bild fungiert als Blickfänger. Da sich die Gesamtbedeutung der Phraseme nicht aus der reinen Addition der Sememe ihrer einzelnen Bestandteile ergibt, sind sie ein geeignetes Mittel, um das Sprachspielerische in der Werbung einzusetzen und außerdem können sie auch mit bildlichen Illustrationen untermauert werden. Die Verknüpfung von Sprache und Bild eröffnet die Möglichkeit von mehreren potenziellen phraseologischen Lesarten, d. h. sowohl die wörtliche als auch die phraseologische Lesart kann aktiviert werden. Die Werbebotschaft ist einprägsam und mittels Phraseme für die Leser leicht verständlich. Aus diesem Grund greifen die Werbetexter oft und bewusst zu Phrasemen, die einerseits feste, fast klischeehafte Strukturen aufweisen, die allgemein bekannt sind und einen möglichst breiten Leserkreis erreichen können. Andererseits erfüllen Phraseme die Anforderung der Werbung nach Originalität.

Obwohl in vielen Forschungsarbeiten darauf hingewiesen wird, dass Phraseme in der Werbung schon relativ gut untersucht seien (vgl. Burger 2015: 180), bestehen in einigen Bereichen noch Forschungslücken. Wenig untersucht sind z.B. Phraseme in der Internet-Werbung und auf Werbeplakaten. Auch Übersetzungen der Werbungen, bei denen u.a. kulturelle Unterschiede eine Wirkung auf die Gestaltung der Werbebotschaft haben, sind bis jetzt wenig untersucht worden.

\section{LITERATUR}

BACHMANN-STEIN, Andrea (2011) Phraseologie und Textmusterstil. H. Lenk, S. Stein (Hg.), Phraseologismen in Textsorten. Hildesheim, Zürich, New York: Georg Olms Verlag. 17-41.

BALSLIEMKE, Petra (1999) Der Kunde ist König! Zur Verwendung von Phraseologismen in der Anzeigenwerbung. R. S. Baur, C. Chlosta, E. Piirainen (Hg.), Wörter in Bildern-Bilder in Wörtern. Beiträge zur Phraseologie und Sprichwortforschung aus dem Westfälischen Arbeitskreis. Baltmannsweiler: Schneider Verlag Hohengehren, 19-46.

BALSLIEMKE, Petra (2001), Da sieht die Welt schon anders aus “: Phraseologismen in der Anzeigenwerbung. Modifikationen und Funktion in Text-Bild-Beziehungen. Baltmannsweiler: Schneider Verlag Hohengehren.

BÁNFFI-BENEDEK, Andrea (2016) Der Einsatz von Somatismen und Kinegrammen im DaF-Unterricht. Eine interkulturelle Annäherung. Germanistische Studien X, $243-254$. 
BASS, Nicole (2003) Phraseologismen und Modifikationen in der Deutschschweizer Anzeigenwerbung 1928-1998. H. Burger, A. Häcki Buhofer, Annelies, G. Greciano (Hg.), Flut von Texten - Vielfalt der Kulturen. Ascona 2001 zur Methodologie und Kulturspezifik der Phraseologie. Baltmannsweiler: Schneider Verlag Hohengehren. 381-390. BURGER, Harald (1991) Phraseologie und Intertextualität. C. Palm (Hg.), Europhras 90. Akten der internationalen Tagung zur germanistischen Phraseologieforschung. Aske/Schweden 12.-15. Juni 1990. Uppsala: Deutsches Institut der Universität Uppsala, 13-27.Burger, Harald (1997) Phraseologie im Kinder- und Jugendbuch. In: R. Wimmer, F.-J. Berens (Hg.), Wortbildung und Phraseologie. Tübingen: G. Narr Verlag, 233-254.

BURGER, Harald (2015) Phraseologie. Eine Einführung am Beispiel des Deutschen. Berlin: Erich Schmidt Verlag.

BURGER, Harald/Annelies BUHOFER/Ambros SIALM (1982) Handbuch der Phraseologie. Berlin, New York: W. de Gruyter.

DOBROVOL'SKIJ, Dmitrij (1980) Zur Dialektik des Begriffs der textbildenden Potenzen von Phraseologismen. Zeitschrift für Phonetik, Sprachwissenschaft und Kommunikationsforschung 33, 690-700.

DOBROVOL 'SKIJ, Dmitrij (1997) ldiome im mentalen Lexikon Ziele und Methoden der kognitivbasierten Phraseologieforschung. Trier: WVT Wissenschaftlicher Verlag.

DUDEN 11 (2002) Redewendungen. Wörterbuch der deutschen Idiomatik. Herausgegeben von der Dudenredaktion. Mannheim, Leipizig, Wien, Zürich: Dudenverlag.

DUDEN (2007) Das große Buch der Zitate und Redewendungen. Mannheim, Leipzig, Wien, Zürich: Dudenverlag.

FIX, Ulla (1997) Kanon und Auflösung des Kanons. Typologische Intertextualität - ein postmodernes Stilmittel? Eine thesenhafte Darstellung. G. Antos, H. Tietz (Hg.), Die Zukunft der Textlinguistik. Traditionen, Transformationen, Trends. Tübingen: Niemeyer, 97-108.

FLEISCHER, Wolfgang (1997) Phraseologie der deutschen Gegenwartssprache. Tübingen: Max Niemeyer Verlag.

FRITZ, Thomas A. (1994) Die Botschaft der Markenartikel: Vertextungsstrategien in der Werbung. Tübingen: Stauffenburg.

GRASSEGER, Hans (1989) Redensarten in der Fernsehwerbung. Zur Struktur und Rezeption von Idiomen in multimedialer Kommunikation. G. Gréciano (Hg.), EUROPHRAS 88. Phraséologie Contrastive. Strassbourg, 141-154.

HEMMI, Andrea (1994) „Es muß wirksam werben, wer nicht will verderben. “ Kontrastive Analyse von Phraseologismen in Anzeigen-, Radio- und Fernsehwerbung. Bern, Berlin: Lang.

JANICH, Nina (2001) Werbesprache. Ein Arbeitsbuch. Tübingen: Gunter Narr Verlag.

JANICH, Nina (2005) Wenn Werbung Sprüche klopft. Phraseologismen in Werbeanzeigen. Der Deutschunterricht 57, 44-53. 
JANICH, Nina (2006) Phraseologismen in der Werbesprache. Verwendungsweisen und methodische Probleme. U. Breuer, I. Hyvärinen (Hg.), Wörter - Verbindungen. Festschrift für Jarmo Korhonen zum 60. Geburtstag. Frankfurt am Main, 175-186.

JESENŠEK, Vida (2021) Beiträge zur deutschen und slowenischen Phraseologie und Parömiologie. Maribor: Univerza v Mariboru, Filozofska fakulteta.

KÜHN, Peter (1985) Phraseologismen und ihr semantischer Mehrwert. Jemandem auf die Finger gucken in einer Bundestagsrede. Sprache und Literatur in Wissenschaft und Unterricht 56; 37-46.

LANGE, Meinolf (1998) Die Verwendung sprachlicher Vorlagen in Texten der Anzeigenwerbung. H. Dietrich (Hg.), Das geht auf keine Kuhhaut. Arbeitsfelder der Phraseologie. Bochum: Universitätsverlag Dr. N. Brockmeyer, 169-198.

LENK, E. H. Hartmut/Stephan, STEIN/ (Hg.), (2011) Phraseologismen in Textsorten. Hildesheim [u.a.]: Olms.

MAJNUSZ-STADNIK, Mariola (2014) Andere Länder, andere Werbestrategien. Phraseologismen und deren Modifikationen in den polnischen und deutschen Werbeanzeigen. Opole: Wydawnictwo Uniwersytetu Opolskiego.

MELLADO BLANCO, Carmen (Hg.) (2008) Beiträge zur Phraseologie aus textueller Sicht. Hamburg: Verlag Dr. Kovač.

PAVIĆ PINTARIĆ, Anita (2015) Deutsche und kroatische Idiome kontrastiv. Eine Analyse von Ausdruck und Funktion. Frankfurt a. M.: Lang.

PFEIFFER, Christian (2016) Frequenz und Funktionen phraseologischer Wendungen in meinungsbetonten Pressetexten (1911-2011). Baltmannsweiler: Schneider Verlag Hohengehren.

PTASHNYK, Stefaniya (2009) Phraseologische Modifikationen und ihre Funktionen im Text: Eine Studie am Beispiel der deutschsprachigen Presse. Baltmannsweiler: Schneider Verlag Hohengehren.

RICHTER-VAPAATALO, Ulrike (2007) Da hatte das Pferd die Nüstern voll. Gebrauch und Funktion von Phraseologie im Kinderbuch. Frankfurt a. M: Lang.

RYPÁČKOVÁ, Lucie (2019) Phraseologismen in der Werbung. H. Menclová, M. Voltrová (Hg.), Experimentierräume in der deutschen Sprachwissenschaft. Pilsen: Westböhmische Universität Pilsen, 143-152.

SABBAN, Annette (2004) Zur Rolle derPhraseme für die Konstituion und Funktion des Textes. K. Steyer, (Hg.), Wortverbindungen - mehr oder weniger fest. Berlin, New York: W. de Gruyter, 238-262.

SABBAN, Annette (2007) Textbildende Potenzen von Phrasemen. H. Burger, D. Dobrovol'skij, P. Kühn, N. R. Norrick (Hg.), Phraseologie: ein internationales Handbuch zeitgenössischer Forschung \# Phraseology: an international handbook of contemporary research 1. Berlin, New York: W. de Gruyter, 237-253.

STEIN, Stephan (1995) Formelhafte Sprache. Untersuchungen zu ihren pragmatischen und kognitiven Funktionen im gegenwärtigen Deutsch. Frankfurt am Main u. a.: Lang 
STÖCKL, Hartmut (2004) Die Sprache im Bild - das Bild in der Sprache: zur Verknüpfung von Sprache und Bild im massenmedialen Text: Konzepte, Theorien, Analysemethoden. Berlin: W. de Gruyter.

UMBORG, Viktoria (2020) Multimodale Verwendung von Phrasemen und Lexemen in der Werbung. In: S. Wahl, E. Ronneberger-Sibold, K. Luttermann (Hg.), Werbung für alle Sinne. Multimodale Kommunikationsstrategien. Wiesbaden: Springer, 43-55. VALENČIČ ARH, Urška (2014) Ein Prinz auf der Erbse. Phraseologie und Übersetzung. Am Beispiel der Kinder- und Jugendliteratur von Christine Nostlinger im Deutschen und Slowenischen. Baltmannsweiler: Schneider Verlag Hohengehren GmbH.

VESALAINEN, Mario (2007) Phrasem in der Werbung. H. Burger, D. Dobrovol'skij, P. Kühn, N. R. Norrick (Hg.), Phraseologie: ein internationales Handbuch zeitgenössischer Forschung \# Phraseology: an international handbook of contemporary research 1. Berlin, New York: W. de Gruyter, 292-302.

\section{ONLINEQUELLEN}

HEGEDÜS-LAMBERT, Claudia (2010) Verfestigte Körpermetaphern und -metonymien in Idiomen mit der Nominalkonstituente Hand, Textes et contextes [Online] 5 | 2010. 20. April 2021. https://preo.u-bourgogne.fr/textesetcontextes/index.php?id=233. REDENSARTEN-INDEX. 2. Mai 2021. https://www.redensarten-index.de/suche.php.

\section{POVZETEK}

\section{„HIER KOMMEN IHRE GRAUEN ZELLEN IN FAHRT“ - FRAZEMI V OGLAŠEVANJU}

Članek se osredotoča na rabo frazeologije v reklamnih oglasih v nemških tiskanih medijih. V večjem delu se navezuje na odnos med frazemi v sliki in besedi. V uvodnem delu se posveti kratkemu pregledu frazeologije kot jezikoslovne discipline ter raziskovanju frazeologije $\mathrm{v}$ reklamnih besedilih, zgradbi reklamnih oglasov in prenovitveni/neprenovitveni rabi frazemov.

$\mathrm{Z}$ opisom izbranih vidikov predstavljamo rezultate analize t. i. semantičnih prenovitev, ki vzpostavljajo določeno razmerje med frazemom v sliki in/ali v besedi. Po tipologiji jezikoslovca Hartmuta Stöckla (2004) smo razmerje med frazemom in sliko najprej opredelili kot latento razmerje ali kot eksplicitno razmerje. Pri latentnem razmerju frazemi zgolj ustvarjajo določeno razmerje med slikovno in jezikovno podobo. Podskupini latentnega razmerja sta vizualno evocirani ali jezikovno evocirani frazemi. Pri eksplicitnem razmerju so frazemi materializirani v sliki in besedi. Frazeme, ki se pojavijo le na enem mestu, smo nadalje opredelili kot punktualno razmerje. V podskupino povezovalnega razmerja smo uvrstili frazeme v reklamnih oglasih, na katere se navezujejo tudi ostali jezikovni elementi. 
Pregled gradiva na podlagi 84 reklamnih besedil je pokazal, da je frazeologija v reklamnih oglasih najpogosteje rabljena eksplicitno in povezovalno. To pomeni, da se frazemi ne pojavljajo izolirano, temveč je besedilo prežeto z ekspresivnim oz. frazeološkim jezikom, ki se navezuje na reklamno sporočilo v slikovnem gradivu in obenem v sloganu ali spremnem besedilu. Prikazani izsledki raziskave pričajo o tem, da so frazemi v reklamnih besedilih zelo pogoste in priljubljene strukture, ki prevzemajo persuasivno funkcijo, ker so razumljivi, konotativni in si jih uporabniki lažje zapomnijo.

Ključne besede: frazeologija, reklamni oglasi, slika, besedilo, modifikacije

\section{ABSTRACT \\ "HIER KOMMEN IHRE GRAUEN ZELLEN IN FAHRT" - PHRASEOLOGICAL UNITS IN ADVERTISEMENTS}

The article deals with the use of the phraseological units in print advertisements in the German print media. The focus is the relations between phraseological units in the texts and images of the advertisements. The introductory part provides a brief overview of phraseology as a linguistic discipline and its role in advertisements, the structure of advertisements and possible modifications of phraseological units. The results of the analysis of semantic modifications showed certain relations between phraseological units in texts and images of the advertisements. According to the typology of Hartmut Stöckl (2004), we first defined the relation between a phraseological unit as a latent or explicit relation. In the latent relation phraseological units create a certain relation between text and image. There are two subgroups of the latent relation: phraseological units, which are visually evoked, and phraseological units, which are textually evoked and expressed. In the explicit relation phraseological units are materialized in image and text. Phraseological units, which appear isolated only in one place, are classified in the subgroup of the punctual relation. In the subgroup of the connected relation are phraseological units, to which other linguistic elements and structures refer. The analysis of 84 print advertisements showed that phraseological units are mostly used explicitly and in a connecting manner. This means phraseological units are not used isolated, but the text is characterized by an expressive or phraseological language that is linked to the advertisement's message in image and text at the same time. The research confirmed that phraseological units are very popular as fixed and idiomatic units in advertisements, because they take on the persuasive functions and represent understandable, connotative and easily memorable structures.

Keywords: phraseology, advertisements, image, text, modification 